\title{
Tratamento de fratura de mandíbula por ferimento de arma de fogo em decorrência
}

\section{de violência doméstica}

\author{
Treatment of jaw fracture due to firearm injury as a result of domestic violence \\ Tratamiento de la fractura de mandíbula debido a uma lesión por arma de fuego como resultado de \\ la violência doméstica
}

Recebido: 28/03/2021 | Revisado: 02/04/2021 | Aceito: 05/04/2021 | Publicado: 16/04/2021

\author{
Kim Henderson Carmo Ribeiro \\ ORCID: https://orcid.org/0000-0003-3325-5897 \\ Universidade Estadual Paulista, Brasil \\ E-mail: kimhenderson@hotmail.fr \\ Ana Carolyna Becher Roseno \\ ORCID: https://orcid.org/0000-0002-1227-5947 \\ Universidade Estadual Paulista, Brasil \\ E-mail: carolbecherr@gmail.com \\ Ana Paula Simões Corrêa \\ ORCID: https://orcid.org/0000-0002-8207-4606 \\ Universidade Estadual Paulista, Brasil \\ E-mail: paulinha_odonto@hotmail.com \\ Francisco Rogério Aguiar de Menezes \\ ORCID: https://orcid.org/0000-0003-0926-9498 \\ Faculdade Ibeco, Brasil \\ E-mail: drfranciscomenezes@gmail.com \\ Mariza Akemi Matsumoto \\ ORCID: https://orcid.org/0000-0001-5389-0105 \\ Universidade Estadual Paulista, Brasil \\ E-mail: mariza.am@gmail.com \\ Ângela Alves de Aguiar Goto \\ ORCID: https://orcid.org/0000-0003-2598-3595 \\ Faculdade Ibeco, Brasil \\ E-mail: clinicafacial@hotmail.com
}

\begin{abstract}
Resumo
$\mathrm{O}$ aumento dos índices de violência interpessoal tem gerado um grande impacto na sociedade mundial, principalmente pelo número de vítimas acometidas por ferimentos causados por projéteis de arma de fogo (PAF). Levando-se em consideração a gravidade desse tipo de lesão, a necessidade de intervenção cirúrgica e o aumento significativo de mulheres vitimizadas, destaca-se a importância do conhecimento, por parte do cirurgião bucomaxilofacial, do procedimento técnico cirúrgico e do acolhimento da vítima. Este trabalho tem como objetivo destacar a problemática social envolvendo o feminicídio e relatar de maneira descritiva um caso, no qual a paciente foi vítima de uma tentativa de homicídio, necessitando, dessa forma, de intervenção cirúrgica para redução e fixação de uma fratura cominutiva em região mandibular. A paciente encontra-se com 8 anos de pós-operatório, apresentando estabilidade da osteossínte e da oclusão, realizando normalmente todas as funções do sistema estomatognático. Mostrando assim sucesso da abordagem cirúrgica escolhida.
\end{abstract}

Palavras-chave: Traumatismos faciais; Fraturas mandibulares; Ferimentos por arma de fogo; Ferimentos e lesões; Violência contra a mulher.

\begin{abstract}
The increase in the rates of interpersonal violence has generated a great impact on world society, mainly due to the number of victims affected by injuries caused by fire gun projectiles. Taking into account the severity of this type of injury, the need for surgical intervention, and the significant increase in victimized women, the importance of knowledge, on the part of the maxillofacial surgeon, of the technical surgical procedure, and the welcoming of the victim is highlighted. This work aims to highlight the social problem involving feminicide and report descriptively a case, in which the patient was the victim of attempted homicide, thus requiring surgical intervention to reduce and fix a comminuted fracture in the mandibular region. The patient is 8 years postoperative, showing osteosynthesis and occlusion stability, normally performing all functions of the stomatognathic system. Thus showing the success of the chosen surgical approach.
\end{abstract}

Keywords: Facial injuries; Mandibular fractures; Wounds, Gunshot; Wounds and injuries; Violence against women. 


\section{Resumen}

El aumento de las tasas de violencia interpersonal ha generado un gran impacto en la sociedad mundial, principalmente por el número de víctimas afectadas por lesiones causadas por proyectiles de armas de fuego (PAF). Teniendo en cuenta la gravedad de este tipo de lesiones, la necesidad de intervención quirúrgica y el aumento significativo de mujeres victimizadas, se destaca la importancia del conocimiento, por parte del cirujano maxilofacial, del procedimiento técnico quirúrgico y la acogida de la víctima. Este trabajo tiene como objetivo resaltar la problemática social que involucra el feminicidio y reportar de manera descriptiva un caso, en el cual la paciente fue víctima de un intento de homicidio, requiriendo así una intervención quirúrgica para la reducción y reparación de una fractura conminuta en la región mandibular. El paciente se encuentra a 8 años de posoperatorio, presentando osteosíntesis y estabilidad de oclusión, desempeñando normalmente todas las funciones del sistema estomatognático. Demostrando así el éxito del abordaje quirúrgico elegido.

Palabras clave: Traumatismos faciales; Fracturas mandibulares; Heridas por arma de fuego; Heridas y traumatismos; Violencia contra la mujer.

\section{Introdução}

A violência é uma temática de grande relevância e, seu consequente aumento, tornou-se um grave problema de saúde pública em vários países, dentre estes, o Brasil (Deslandes et al., 2000). A violência interpessoal é responsável por um elevado índice de morbidade e mortalidade, constituindo, dessa forma, um importante problema de saúde pública (Stöckl et al., 2013).

Somado a este índice crescente, chama a atenção, o número de mulheres vítimas de homicídio. No Brasil, essas violências constituem um problema de extrema importância, tendo em vista que, mundialmente, o país ocupa o $5^{\circ}$ maior risco de óbitos por homicídios femininos (Nowak, 2012). Quando analisadas as principais regiões do corpo atingidas durante as agressões, observa-se que as vítimas sofrem mais lesões na região de cabeça e pescoço (Orellana et al., 2019).

$\mathrm{O}$ aumento crescente da violência interpessoal afetou o mundo como um todo, e as lesões por projéteis de arma de fogo (PAF) afetaram a população (Gonzalez-Perez et al., 2017). Lesões por PAF são consideradas, dentro do segmento de trauma facial, o segundo fator nas causas de morte, sendo superado apenas pelos acidentes automobilísticos (Shvyrkov, 2013). Os ferimentos causados por PAF apresentam alta incidência nas regiões de cabeça e pescoço (Oliveira et al., 2007), e é apontado como a segunda principal causa dos feminicídios (Caicedo et al., 2019).

A presença de uma arma de fogo em atos violentos aumenta a probabilidade de morte e de ferimentos graves. Além de altamente letais, as armas de fogo são responsáveis por $29 \%$ das internações hospitalares por agressões e tentativas de suicídio no Brasil (Ribeiro et al., 2017). Nos casos de lesões acometidas por disparos de arma de fogo, a mandíbula é o osso facial mais acometido (Pereira et al., 2012). Estes traumas, normalmente, resultam em fraturas cominutivas com pequenos e múltiplos fragmentos ósseos, podendo estar relacionados com a avulsão dos tecidos duros e moles e com a desvitalização de tecidos próximos e fraturas de estruturas ósseas (Wulkan et al., 2005), sendo responsáveis por diversas complicações do sistema estomatognático como um todo (Zachariades et al., 2006), além de apresentar um alto risco de infecção (Luz et al., 2013) o que exige cuidados pré, trans e pós- operatórios destinados à prevenção de quadros infecciosos (Motamedi, 2011).

Nesse contexto, com uma investigação aprofundada da literatura, pretendemos trazer subsídios e elencar pontos importantes para o tratamento de lesões por PAF apresentando um relato de caso sobre o assunto.

\section{Metodologia}

Este trabalho visa relatar um caso de forma descritiva e qualitativa segundo Pereira et al. (2018), com o objetivo de expor o tratamento cirúrgico de uma fratura cominutiva de mandíbula por projétil de arma de fogo, após tentativa de feminicídio, detalhando assim, a abordagem escolhida suportada pela literatura (Ellis et al., 2003), além de apresentar as singularidades, possíveis sequelas e tipos de tratamento para esse tipo de lesão. A exposição do caso foi autorizado pela paciente por meio do Termo de Consentimento Livre e Esclarecido (TCLE), elaborado em linguagem de fácil compreensão 
para que estivesse ciente do destino das informações pessoais recolhidas, bem como os riscos e benefícios associados, além de enviado ao sistema CEP/CONEP.

\section{Relato de Caso}

Paciente de 51 anos, sexo feminino, apresentou uma fratura cominutiva em região de corpo, ângulo e ramo mandibular esquerdo, comprovada pela tomografia computadoriza (T.C), após ser vítima de uma tentativa de homicídio. Durante a avaliação clínica extraoral foi observada presença de zona de chamuscamento em volta do orifício de entrada do projétil, em região submandibular esquerda, sem a presença de um orifício de saída, edema importante compatível com a lesão e assimetria facial. Somado a isto, a paciente possuía paralisia facial ao sorrir e relatava parestesia em toda hemiface inferior esquerda e língua. Na avaliação intraoral, observou-se maloclusão, limitação de abertura bucal, desvio mandibular e fratura exposta ao meio bucal. Na T.C observou-se fratura cominutiva em região de corpo, ângulo e ramo mandibular esquerdo (Imagem 1A e B). Em seguida, sob anestesia local, foram colocados 6 parafusos de bloqueio e realizado bloqueio maxilomandibular (BMM) com uso de fio de aço afim de se estabilizar a fratura e oclusão, e colocada uma sonda nasoentérica (Imagem $1 \mathrm{C}$ e D).

Imagem 1 - Reconstrução tridimensional da tomografia computadorizada pré-operatória. A: Vista frontal sendo observada maloclusão e fratura cominutiva em corpo e ângulo esquerdo. B: Vista lateral esquerda e presença de fratura vertical de ramo. C: Vista frontal após BMM e presença de sonda nasoentérica. D: Vista lateral esquerda após BMM e presença de sonda nasoentérica.

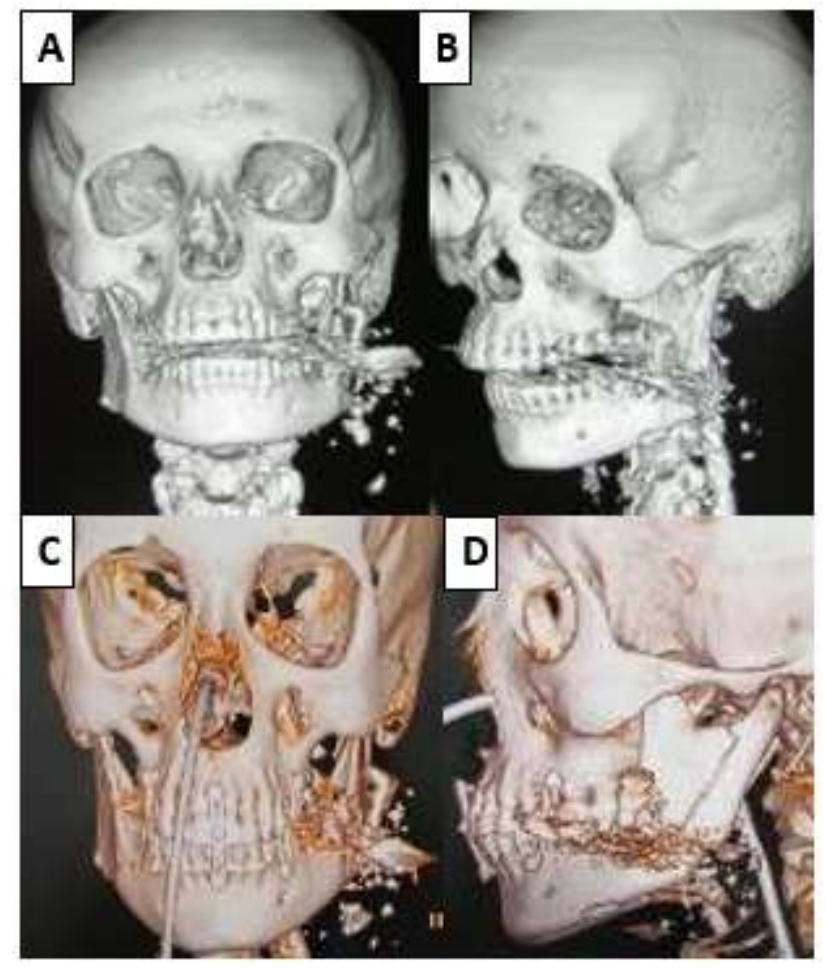

Fonte: Arquivo Pessoal.

A abordagem cirúrgica acerca das fraturas foi feita sob anestesia geral, em âmbito hospitalar, onde, primeiramente, foi realizada uma abundante desinfecção do campo cirúrgico, com pvpi, realizada uma incisão retromandibular e divulsão por planos até exposição completa dos cotos ósseos fraturados. O projétil foi removido durante a cirurgia, juntamente com todos os 
estilhaços ósseos, acarretando um defeito ósseo em região de ângulo esquerdo, deixando um gap ósseo (Imagem 2A). Em seguida, duas placas em "X" do sistema de $2.0 \mathrm{~mm}$ foram instaladas, com o intuito de simplificar e reduzir a fratura vertical em região de ramo mandibular e, posteriormente, uma placa de reconstrução englobando as regiões de corpo e ramo do sistema de $2.4 \mathrm{~mm}$ foi instalada associada a parafusos bicorticais, como demonstra a Imagem 2B. Por fim, os tecidos foram debridados e realizada sutura por planos, retratada na Imagem 2C. A paciente permaneceu internada por 72 horas, e após alta hospitalar, recebeu assistência em domicílio para acompanhamento de antibioticoterapia, permanecendo sob cuidados do infectologista devido ao alto risco de infecção deste tipo de lesão, tornando o acompanhamento a longo prazo indispensável.

Imagem 2 - Imagens do trans operatório. A: Exposição dos cotos e gap ósseo. B: Fixação da fratura com placa em X do sistema 2.0 e de reconstrução $2.4 \mathrm{~mm}$. C: Vista lateral da paciente no pós-operatório imediato e sutura.

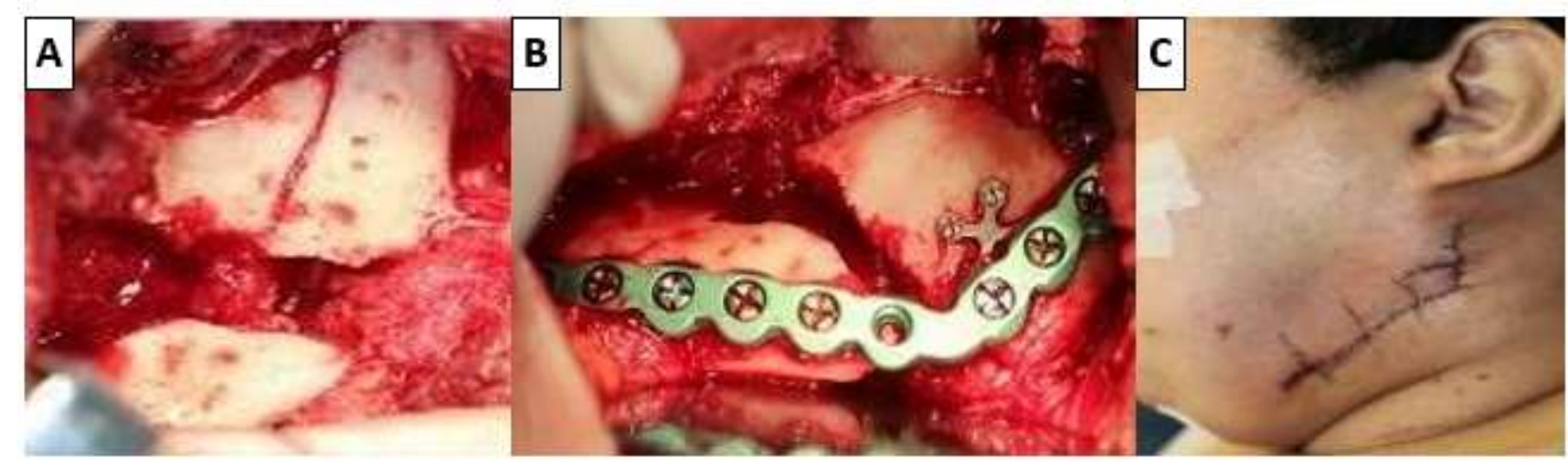

Fonte: Arquivo Pessoal.

Aos 5 anos de pós-operatório, observa-se presença de leve assimetria facial mantida (Imagem 3A) e boa cicatrização no local do acesso (Imagem 3B). Já nas reconstruções tridimensionais da tomografia computadorizada, constata-se manutenção da osteossíntese, e boa estabilidade oclusal (Imagem 3C e 3D).

Atualmente, a paciente encontra-se com 8 anos de pós-operatório, apresentando melhora na simetria facial, leve limitação abertura bucal, nega sintomatologia dolorosa, realiza, normalmente, as funções de mastigação, manteve parestesia em língua (lado esquerdo) e apresenta estabilidade da osteosíntese e oclusal observada pela radiografia panorâmica recente (Imagem 3E). 
Imagem 3 - Imagens de cinco anos de pós-operatório. A: Vista frontal. B: Vista lateral esquerda e cicatrização. C: TC vista frontal da reconstrução 3D. D: Vista lateral esquerda da reconstrução 3D. E: Radiografia panorâmica controle de 8 anos pósoperatório.

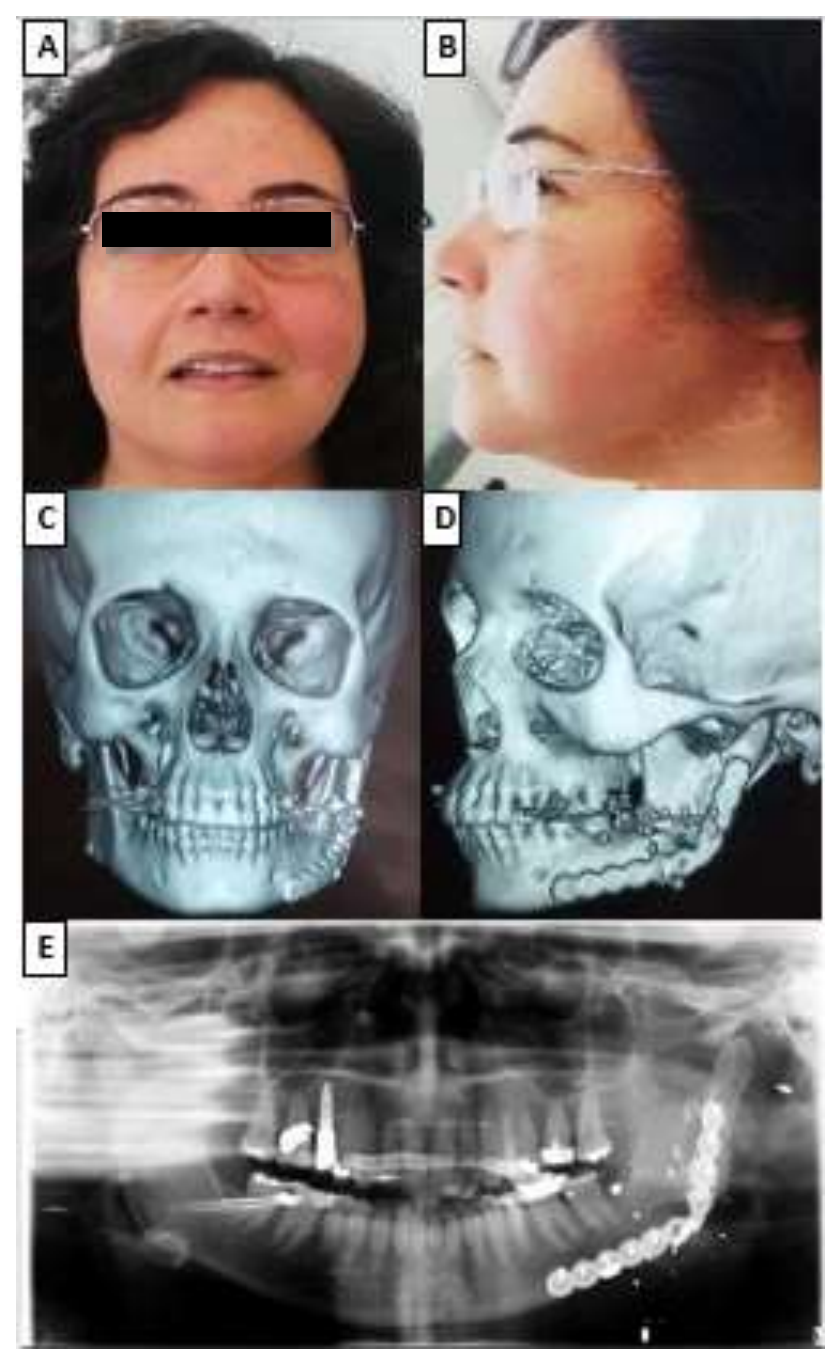

Fonte: Arquivo Pessoal.

\section{Discussão}

A violência é reconhecida mundialmente como relevante problema social e de saúde pública, que se caracteriza como qualquer comportamento que cause intencionalmente danos graves ou algum tipo de prejuízo a si mesmo ou a outro indivíduo, podendo comprometer a saúde e qualidade de vida (Souza et al., 2016).

Um importante fator que contribui para os altos níveis de violência é a posse de arma de fogo (Gonzalez-Perez et al., 2017). Ademais, vale pontuar que, entre os atendidos por lesões provocadas por armas de fogo, predominam as vítimas por agressões (79,7\%), o que configuram tentativas de homicídio (Ribeiro et al., 2017). É importante ressaltar que em locais onde há grande circulação de armas de fogo, há maior proporção de homicídios femininos (Alvazzi, 2011). Nos últimos 5 anos, ocorreu um aumento de $25 \%$ nos homicídios de mulheres por arma de fogo dentro das residências, por sua vez, parece refletir o crescimento na difusão das armas (Cerqueira et al., 2019).

O caso apresenta relevância por levantar dois pontos importantes que devem ser levados em consideração: traumas faciais acometidos por armas de fogo e principais regiões afetadas em situações de feminicídio. Os ferimentos causados por projéteis de arma de fogo apresentam elevada incidência na região de cabeça/face e pescoço (Oliveira et al., 2007), sendo três 
vezes mais frequentes em casos de feminicídio (Alvis et al., 2016). Dentre os locais mais afetados, aponta-se a mandíbula, especialmente na região de corpo (Alper et al., 1998), sendo observado em nosso caso, em que o corpo mandibular foi a região com maior presença de estilhaços ósseos, seguido de ângulo e ramo.

Vários fatores são levados em consideração em ferimentos por PAF, como por exemplo: forma, tamanho, composição e velocidade dos projéteis. Soma-se a eles, o ângulo de penetração e o movimento, que também afetam a magnitude do trauma (Aarabi et al., 2003). Quanto ao caso apresentado, a presença de zona de chamuscamento bem marcada circundando o orifício de entrada, pode caracterizar um disparo a curta distância.

As fraturas ósseas podem ser classificadas, de acordo com a condição dos fragmentos ósseos como: simples, compostas, cominutivas, galho-verde ou impactada (Kruger, 1979). As fraturas ocasionadas por arma de fogo, na maioria dos casos, enquadram-se como fraturas cominutivas (Ellis et al., 2003). No presente caso, a paciente vítima de tentativa de feminicídio por seu ex-parceiro, apresentou uma lesão cominutiva. No que se refere ao tratamento, em casos de fraturas em ossos faciais, causados por armas de fogo, opta-se pela redução e fixação dos cotos ósseos, objetivando reduzir as chances de complicações (Motamedi, 2012). A escolha dessa conduta faz com que a cicatrização da ferida óssea não seja interrompida para a realização de uma nova cirurgia subsequente e, também, não submete o paciente a um segundo estresse cirúrgico (Luz et al., 2013), sendo realizado no caso exposto, por meio de placa de reconstrução e parafusos bicorticais. A escolha da placa $2.4 \mathrm{~mm}$ é bastante defendida na literatura para a fixação de fraturas cominutivas com perda de substância, e consequente formação de gap entre os cotos proporcionando melhor estabilização dos segmentos (Pereira et al., 2012).

Tão importante quanto a determinação da técnica empregada pelo cirurgião e da escolha dos métodos fixadores, temse a necessidade de estabelecer uma boa terapia antibiótica para pacientes vítimas de fraturas causadas por PAF. A literatura demonstra que a administração de um adequado esquema medicamentoso reduz significativamente o número de infecções no pós-operatório (Von et al., 2011).

No presente caso, levando-se em consideração que se tratava de uma fratura exposta com íntimo envolvimento entre o meio intraoral e extraoral, realizou-se profilaxia antibiótica e acompanhamento médico multidisciplinar durante sua internação, ressaltando-se o papel do infectologista responsável pelo controle da infecção, muito comum nesse tipo de ferimento, além de um acompanhamento clínico por anos da sua vida.

\section{Conclusão}

Conclui-se que com aumento dos casos de violência, em especial contra as mulheres, e levando-se em consideração os locais mais acometidos por esses atos, destaca-se a importância do conhecimento técnico cirúrgico assim como dos materiais fixadores a serem empregados para ideal manejo da vítima, além da adequada conduta pós-operatória por parte do cirurgião bucomaxilofacial. Com esse estudo, é possível constatar que as fraturas acometidas por PAF estão cada vez mais frequentes nos postos de atendimento exigindo que o cirurgião esteja apto a acolher e desempenhar sua conduta de forma a garantir o bem-estar das pacientes e sua saudável recuperação.

No entanto, acredita-se que os seguintes pontos são interessantes de serem explorados em estudos posteriores: análises e comparações entre diferentes metodologias de tratamento em fraturas PAF ao propósito de produzir informações suficientes para definição de um protocolo.

\section{Referências}

Aarabi B. (1995). Management of traumatic aneurysms caused by high-velocity missile head wounds. Neurosurgery clinics of North America, 6(4), 775-797.

Alper, M., Totan, S., Cankayali, R., \& Songür, E. (1998). Gunshot wounds of the face in attempted suicide patients. Journal of oral and maxillofacial surgery: official journal of the American Association of Oral and Maxillofacial Surgeons, 56(8), 930-934. https://doi.org/10.1016/s0278-2391(98)90652-8 
Alvazzi A. (2011). When the victim is a woman. In: Geneva Declaration. www.genevadeclaration.org/fileadmin/docs/GBAV2/GBAV2011_CH4.pdf.

Alvis-Miranda, H. R., Rubiano, A., Agrawal, A., Rojas, A., Moscote-Salazar, L. R., Satyarthee, G. D., Calderon-Miranda, W. G., Hernandez, N. E., \& Zabaleta-Churio, N. (2016). Craniocerebral Gunshot Injuries; A Review of the Current Literature. Bulletin of emergency and trauma, 4(2), 65-74.

Caicedo-Roa, M., Cordeiro, R. C., Martins, A. C. A., \& de Faria, P. H. (2019). Femicídios na cidade de Campinas, São Paulo, Brasil. Cadernos de Saúde Pública, 35(6), e00110718. https://dx.doi.org/10.1590/0102-311x00110718

Cerqueira, D., Bueno, S., Lima, R. S., Neme, C., Ferreira, H., Alves, P. P., Marques, D., Reis, M., Cypriano, O., Sobral, I., Pacheco, D., Lins, G., \& Armstrong, K. (2019). Atlas da Violência 2019. http://repositorio.ipea.gov.br/handle/11058/9489.

Deslandes, S. F., Gomes, R., \& da Silva, C. M. F. (2000). Caracterização dos casos de violência doméstica contra a mulher atendidos em dois hospitais públicos do Rio de Janeiro. Cadernos de Saúde Pública, 16(1), 129-137. https://doi.org/10.1590/S0102-311X2000000100013

Ellis III E., Muniz O., \& Anand K. (2003). Treatment considerations for comminuted mandibular fractures. J Oral Maxillofac Surg.; 61: 861-70. http://dx.doi.org/10.1016/S0278-2391(03)00249-0

González-Pérez, G. J., Vega-López, M. G., \& Flores-Villavicencio, M. E. (2017). El incremento de la mortalidad por armas de fuego y su relación con el estancamiento de la esperanza de vida en México. Ciência \& Saúde Coletiva, 22(9), 2861-2872. https://doi.org/10.1590/1413-81232017229.21902016

Kruger G. O. (1979). Fraturas de Mandíbula. In: Kruger GO. Cirurgia bucal e maxilo-facial: Guanabara Koogan; p. 253-286.

Luz, J. G., Moraes, R. B., D'Ávila, R. P., \& Yamamoto, M. K. (2013). Factors contributing to the surgical retreatment of mandibular fractures. Brazilian oral research, 27(3), 258-265. https://doi.org/10.1590/S1806-83242013005000007

Motamedi M. H. (2011). Management of firearm injuries to the facial skeleton: Outcomes from early primary intervention. Journal of emergencies, trauma, and shock, 4(2), 212-216. https://doi.org/10.4103/0974-2700.82208

Nowak, M. (2012). Femicide: a global problem. Small Arms Survey Research Notes. Geneva. http://www.smallarmssurvey.org/fileadmin/docs/HResearch_Notes/SAS-Research-Note-14.pdf.

Oikarinen, K., Ignatius, E., Kauppi, H., \& Silvennoinen, U. (1993). Mandibular fractures in northern Finland in the 1980s--a 10-year study. The British journal of oral \& maxillofacial surgery, 31(1), 23-27. https://doi.org/10.1016/0266-4356(93)90092-b

de Oliveira, D. M., Vasconcellos, R. J., Laureano Filho, J. R., \& Cypriano, R. V. (2007). Fracture of the coronoid and pterygoid processes by firearms: case report. Brazilian dental journal, 18(2), 168-170. https://doi.org/10.1590/s0103-64402007000200016

Orellana, J. D. Y., da Cunha, G. M., Marrero, L, Horta, B. L., \& Leite, I. C. (2019). Urban violence and risk factors for femicide in the Brazilian Amazon. Cadernos de Saúde Pública, 35(8), e00230418. https://doi.org/10.1590/0102-311x00230418

Pereira, A. S., Shitsuka, D. M., Parreira, F. J., \& Shitsuka, R. (2018). Metodologia da Pesquisa Científica. UFSM. https://repositorio.ufsm.br/bitstream/handle/1/15824/Lic_Computacao_Metodologia-Pesquisa -Cientifica.pdf?sequence=1.

Pereira, C. C., Letícia Dos Santos, P, Jardim, E. C, Júnior, I. R., Shinohara, E. H., \& Araujo, M. M. (2012). The Use of 2.4-mm Locking Plate System in Treating Comminuted Mandibular Fracture by Firearm. Craniomaxillofacial trauma \& reconstruction, 5(2), 91-96. https://doi.org/10.1055/s-0032-1313364

Ribeiro, A. P., de Souza, E. R., \& de Sousa, C. A. M (2017). Injuries caused by firearms treated at Brazilian urgent and emergency healthcare services. Ciência \& Saúde Coletiva, 22(9), 2851-2860. https://doi.org/10.1590/1413-81232017229.16492017

Shvyrkov M. B. (2013). Facial gunshot wound debridement: debridement of facial soft tissue gunshot wounds. Journal of cranio-maxillo-facial surgery : official publication of the European Association for Cranio-Maxillo-Facial Surgery, 41(1), e8-16. https://doi.org/10.1016/j.jcms.2012.04.001

Stöckl, H., Devries, K., Rotstein, A., Abrahams, N., Campbell, J., Watts, C., \& Moreno, C. G. (2013). The global prevalence of intimate partner homicide: a systematic review. Lancet (London, England), 382(9895), 859-865. https://doi.org/10.1016/S0140-6736(13)61030-2

von See, C., Rana, M., Stoetzer, M., Wilker, C., Rücker, M., \& Gellrich, N. C. (2011). A new model for the characterization of infection risk in gun shot injuries: technology, principal consideration and clinical implementation. Head \& face medicine, 7, 18. https://doi.org/10.1186/1746-160X-7-18

Wulkan, M, Parreira J. G., \& Botter, D. A. (2005). Epidemiologia do trauma facial. Revista da Associação Médica Brasileira,51(5), 290295. https://doi.org/10.1590/S0104-42302005000500022

Zachariades, N., Mezitis, M., Mourouzis, C., Papadakis, D., \& Spanou, A. (2006). Fractures of the mandibular condyle: a review of 466 cases. Literature review, reflections on treatment and proposals. Journal of cranio-maxillo-facial surgery: official publication of the European Association for Cranio-MaxilloFacial Surgery, 34(7), 421-432. https://doi.org/10.1016/j.jcms.2006.07.854 